\title{
Defining the Indications and Levels of Erector Spinae Plane Block in Pediatric Patients: A Retrospective Study of Our Current Experience
}

\author{
Can Aksu ${ }^{1}$, Yavuz Gurkan ${ }^{2}$ \\ 1. Anesthesiology, Kocaeli University, Kocaeli, TUR 2. Anesthesiology, Koç University, İstanbul, TUR
}

Corresponding author: Can Aksu, dr.aksu@gmail.com

\section{Abstract}

\section{Aim}

The primary aim of this study was to evaluate the indications, effectiveness, application levels, and local anesthetic (LA) dosages used in erector spinae plane block (ESPB) in pediatric patients based on our clinical data. The secondary aim was to compare previously reported pediatric ESPBs with our data and to prepare a mini-guide for future clinical applications.

\section{Materials and methods}

One hundred and forty-one pediatric patients who received ESPB and were operated by the Department of Pediatric Surgery were included in this retrospective observational study. ESPB is routinely performed with $0.5 \mathrm{ml} / \mathrm{kg} 0.25 \%$ bupivacaine (max $20 \mathrm{ml}$ ). Demographic data and the type of surgery were recorded. Face, Legs, Activity, Cry, and Consolability (FLACC) or Numeric Rating Scale (NRS) scores, analgesic requirements, and the type of analgesic administered at postoperative period were recorded.

\section{Results}

ESPB was applied using three different techniques, the classic approach, the transverse approach, and the Aksu approach. Unilateral ESPB was performed on 112 patients, while 29 received a bilateral block. ESPB used for 13 different indications.

\section{Conclusion}

ESPB is a relatively safe and effective procedure for achieving opioid-free postoperative analgesia in many different surgical procedures in pediatric patients.

Received 07/31/2019

Review began 08/04/2019 Review ended 08/07/2019 Published 08/08/2019

\section{() Copyright 2019}

Aksu et al. This is an open access article distributed under the terms of the Creative Commons Attribution License CC-BY 3.0., which permits unrestricted use, distribution, and reproduction in any medium, provided the original author and source are credited.
Categories: Anesthesiology, Pain Management, Pediatric Surgery

Keywords: erector spinae plane block, postoperative pain, pediatric anesthesia, postoperative analgesia, ultrasound

\section{Introduction}

The erector spinae plane block (ESPB) was first described by Forero et al. [1] in 2016 and has since become a trending topic among clinicians. However, the problem with ESPB is that there still is a lack of knowledge concerning its mechanism of action, and clinical reports/studies have produced inconsistent results regarding local anesthetic (LA) distribution [2-4]. However, although the mechanism of action and drug distribution have not been clearly elucidated, the clinical significance of this block is undeniable.

There have been a few randomized prospective studies of ESPB in terms of its clinical effectiveness, but all, with one exception, have involved adults [5-7]. Pediatric case reports are also limited compared to adults [824]. We have been applying ESPB almost since it was first described. However, ESPB applications for pediatric patients in our clinic commenced following a learning curve. Nonetheless, we have to date performed numerous ESPB applications in pediatric patients with different indications.

Various reviews and reports have investigated the indications for ESPB in adults, but to the best of our knowledge, there is no published definitive study for pediatric patients. We have been using standardized methods for perioperative analgesia management of pediatric patients in our clinic, and the data are routinely preserved. The primary aim of this study was to evaluate the indications, effectiveness, application levels, and LA dosages used in ESPB in pediatric patients based on our clinical data. The secondary aim was to compare previously reported pediatric ESPBs with our data and to prepare a mini-guide for future clinical applications.

\section{Materials And Methods}


This retrospective cohort study was conducted after receipt of approval from the Local Ethics Committee (GOKAEK 2019/85) and registration with clinicaltrials.org (NCT03906019). The study was conducted in accordance with the Declaration of Helsinki. We reviewed all medical records of patients operated by the Department of Pediatric Surgery between September 1, 2017 and March 1, 2019, and undergoing ESPB as a part of postoperative analgesia management. Following the establishment of the patient population, other requested data elements were retrieved in a series of further queries and recorded on IBM SPSS for Windows ${ }^{\circledR}$ version 20.0 (SPSS, Chicago, IL, USA) software for subsequent analysis. Collected data included demographic information, patient position for the block, indication/surgery type, level of ESPB, unilateral or bilateral application, the total volume applied, additional analgesic use, kind of analgesic used, if applicable, and any additional descriptive data the patient provided during follow-up.

Our clinic has a standard protocol for pediatric surgeries for both anesthesia and pain management. Every patient under the age of 15 years receives ESPB under general anesthesia before the start of surgery, and patients older than 15 years were asked whether or not they are willing to undergo awake block application. We use $0.25 \%$ bupivacaine as the LA for all block applications. The volume of LA administered for a unilateral block is $0.5 \mathrm{ml} / \mathrm{kg}$ (max $20 \mathrm{ml}$ ). Every patient receives paracetamol $15 \mathrm{mg} / \mathrm{kg}$ iv routinely at the end of surgery. For the postoperative follow-up, except in day-case surgery, paracetamol $15 \mathrm{mg} / \mathrm{kg}$ is routinely administered to every patient under the age of one year every six hours for the first postoperative day. In subsequent days, Face, Legs, Activity, Cry, and Consolability (FLACC) or Numeric Rating Scale (NRS) scores are used for administering any analgesic drugs. There is no routine analgesic regimen for children over one year, although additional analgesic drug requirements are decided based on FLACC or NRS scores for the entire postoperative period. FLACC is the preferred scoring system for patients up to seven years of age, while the NRS score is the accepted pain evaluation method for patients older than seven. Paracetamol, non-steroidal anti-inflammatory drugs or tramadol are used depending on pain severity. A pediatric pain nurse is responsible for all pain evaluations, recording pain score data, and administering analgesic drugs.

For day case surgeries, patients are discharged home after a six-hour follow-up period. In order to assess pain and administer analgesic drugs at home, pain-scoring systems are taught to parents, who are instructed to administer $15 \mathrm{mg} / \mathrm{kg}$ oral acetaminophen as the first choice rescue analgesia and $7 \mathrm{mg} / \mathrm{kg}$ oral ibuprofen if the pain is severe. Analgesic requirements and the type of analgesic administered were questioned and recorded at postoperative follow-up visits for all patients. The acute pain team routinely maintains all data. Due to this standardization, the results of this study represent retrospective analyses of routinely collected data.

\section{Results}

Data for 141 pediatric patients receiving ESPB, 44 girls and 97 boys, were included in the study. The mean age of the patients was $4.95 \pm 4.09$ years ( $\min 0.25-\max 17$ ), and their mean weight was $21.58 \pm 14.86 \mathrm{~kg}$ (min 4 - max 68). The American Society of Anesthesiology (ASA) physical status scores were '1' for 127 patients and ' 2 ' for 14 . Mean operative time was $65.32 \pm 35.91 \min (\min 30-\max 300)$.

ESPB was applied using three different techniques: the classic approach as seen in Figure 1, the transverse approach [22] as seen in Figure 2, and the Aksu approach [20] as seen in Figure 3.

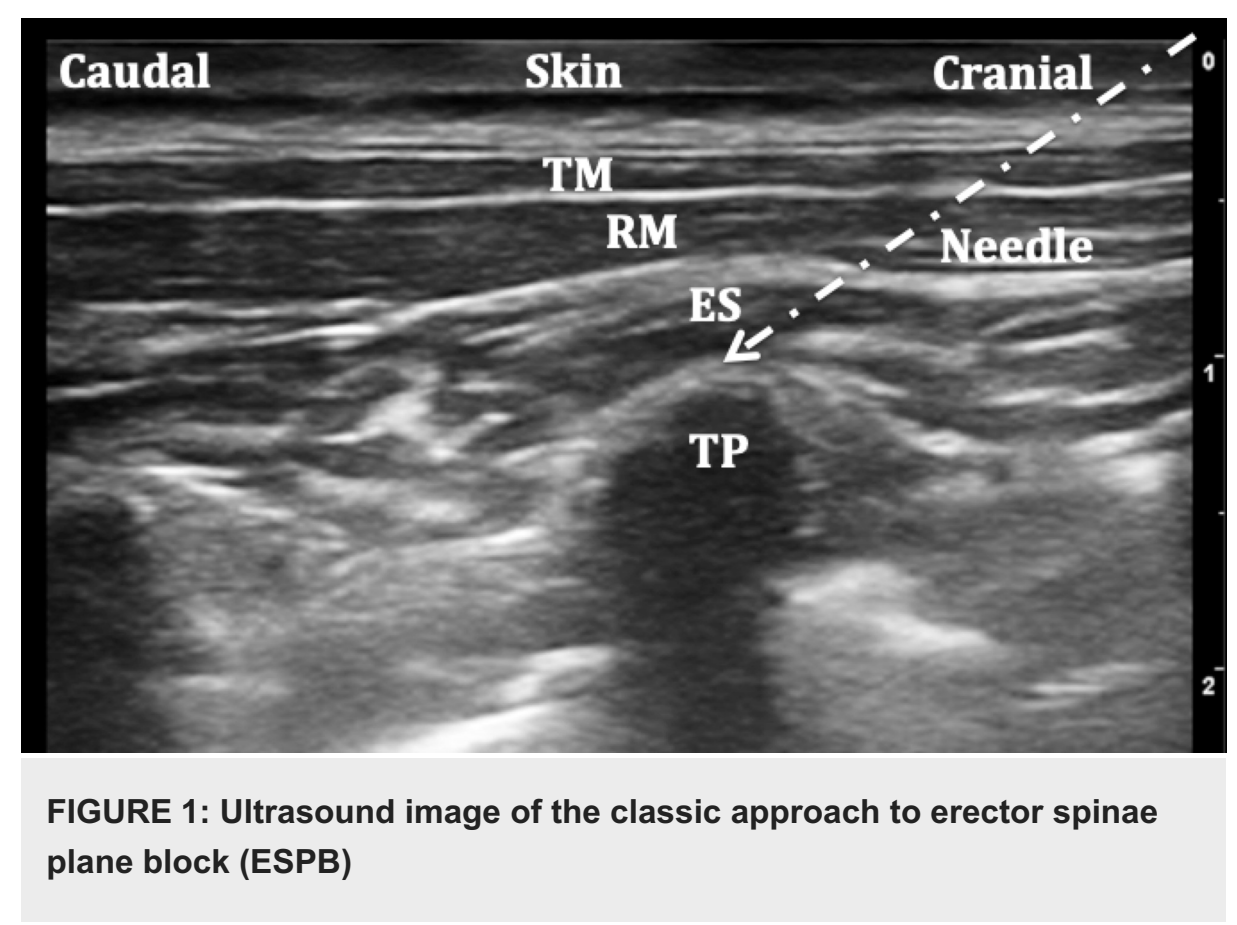




\section{Cureus}

TM: Trapezius muscle, RM: Rhomboid muscle, ES: Erector spinae, TP: Transverse process.

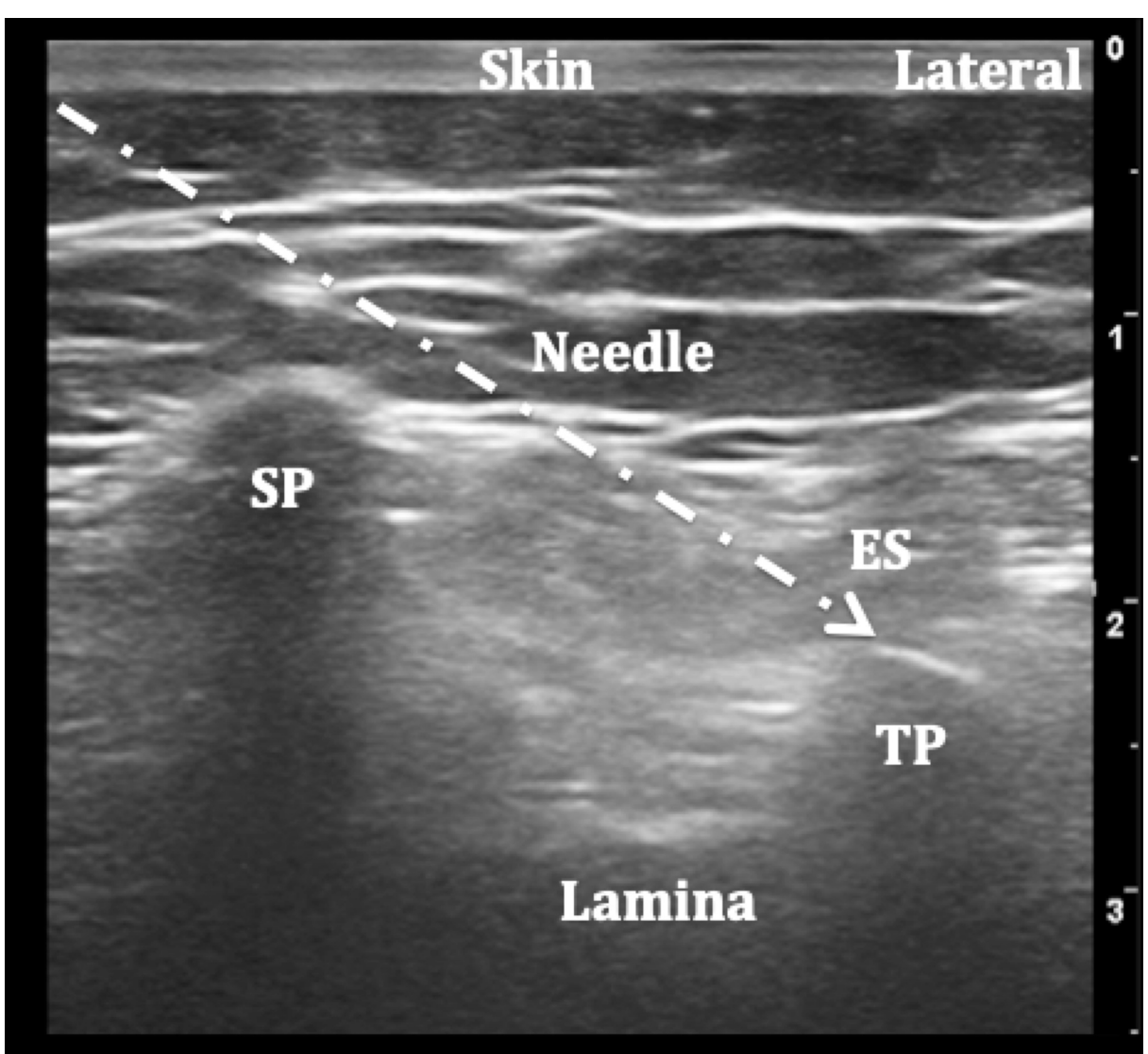

FIGURE 2: UItrasound image of the transverse approach to erector spinae plane block (ESPB)

SP: Spinous process, ES: Erector spinae, TP: Transverse process. 


\section{Cureus}

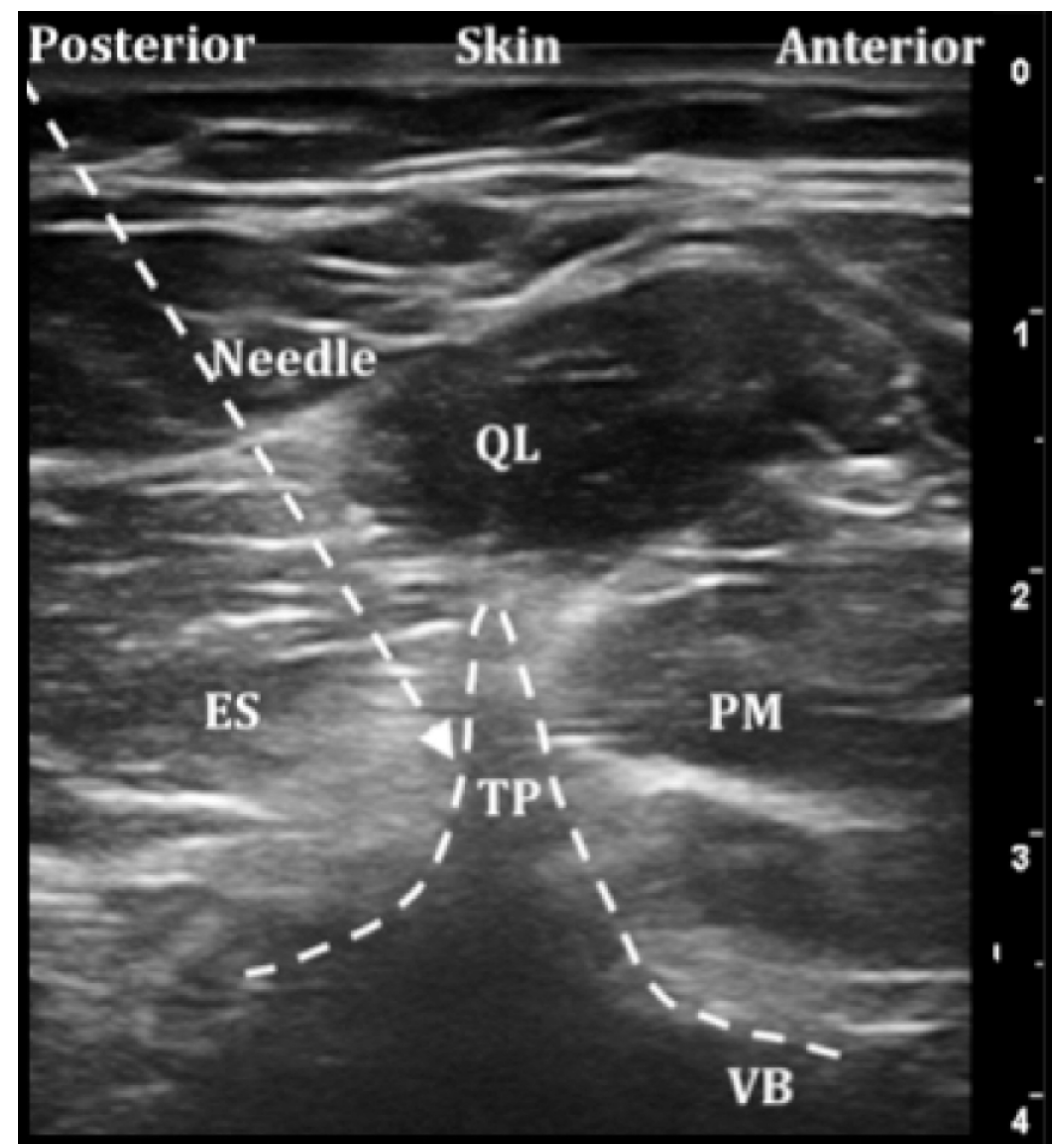

FIGURE 3: Ultrasound image of the Aksu approach to erector spinae plane block (ESPB) at lumbar levels

ES: Erector spinae, QL: Quadratus lumborum muscle, PM: Psoas muscle, TP: Transverse process, VB: Vertebral body.

Ninety-seven patients underwent ESPB in the prone position and 44 in the lateral decubitus position, the Aksu approach [20] being applied to 35 of these.

Unilateral ESPB was performed on 112 patients, while 29 received a bilateral block. Five of these bilaterally blocked patients were received a sacral ESPB, which was a newly defined level for this block application. Four of these patients were operated for circumcision and one for anoplasty.

ESPB was used for 13 different indications. The mean parental or patient satisfaction score was 9.2 \pm 0.65 . Indications, block application levels, numbers of patients requiring rescue analgesia, and types of rescue analgesia are shown in Table 1. 


\section{Cureus}

\begin{tabular}{|c|c|c|c|}
\hline & $\begin{array}{l}\text { Number of } \\
\text { Patients }\end{array}$ & Application Level & $\begin{array}{l}\text { Number of Patients Requiring Rescue Analgesia } \\
\text { (Paracetamol / NSAID) }\end{array}$ \\
\hline Inguinal hernia repair & 50 & $\begin{array}{l}\text { L1-L2 /Aksu } \\
\text { approach }\end{array}$ & $5 / 0$ \\
\hline Orchiopexy & 47 & $\begin{array}{l}\text { L1-L2 /Aksu } \\
\text { approach }\end{array}$ & $5 / 1$ \\
\hline Hydrocelectomy & 10 & $\begin{array}{l}\text { L1-L2 /Aksu } \\
\text { approach }\end{array}$ & $1 / 0$ \\
\hline $\begin{array}{l}\text { Laparoscopic } \\
\text { cholecystectomy }\end{array}$ & 6 & T7-T8 & $1 / 1$ \\
\hline Nephrectomy & 6 & T10-T12 & $2 / 0$ \\
\hline Varicocelectomy & 4 & T11-T12 & $0 / 1$ \\
\hline Ovarian surgery & 6 & T10 & $1 / 3$ \\
\hline Thoracic surgery & 1 & T4 & $1 / 0$ \\
\hline Breast surgery & 2 & T4 & $0 / 1$ \\
\hline Anoplasty & 1 & S4 & $0 / 0$ \\
\hline Pyeloplasty & 2 & T9-T10 & $1 / 0$ \\
\hline Circumcision & 4 & S3-S4 & $0 / 0$ \\
\hline Colostomy surgery & 2 & T10-T11 & $2 / 0$ \\
\hline
\end{tabular}

TABLE 1: Indications and levels of erector spinae plane block (ESPB) and rescue analgesic use NSAID: nonsteroidal anti-inflammatory drugs.

We determined only one exception to the LA dosage. For the patient that underwent thoracotomy, unilateral ESPB was performed from the T4 level with $1 \mathrm{ml} / \mathrm{kg} 0.25 \%$ bupivacaine. No block-related complications were observed in any patients.

\section{Discussion}

This retrospective cohort study investigated ESPB applications for 141 patients with 13 different indications, of which eight were thoracic, three were lumbar, and two were at the sacral level. The use of three different novel techniques reported in this study.

Although ESPB is a relatively new regional anesthesia technique, it has attracted significant interest from clinicians due to its clinical effectiveness and ease of administration. There are increasing reports of its use for different indications. As for many other regional techniques, reports of its use in pediatric patients are still limited and are also restricted to case reports, except for Aksu et al. [7].

Indications for ESPB for postoperative analgesia in pediatric surgeries to date include thoracotomy [8-9], video-assisted thoracoscopic surgery [10], pectus excavatum/carinatum [11], vascular ring repair [12], sternotomy [13], major abdominal surgery [14], laparoscopic cholecystectomy [15-16], nephrectomy [17], pyeloplasty [18], inguinal hernia repair [7,19-21], orchiopexy [7], hydrocelectomy [7], varicocelectomy [22] and hip surgery [23]. There has also been one report of its use in pain management in pediatric palliative care [24]. We have achieved successful applications in five new indications for pediatric patients, ovarian surgery, breast surgery, anoplasty, colostomy, and circumcision. As this interest in ESPB continues to grow, it may be anticipated that many more indications will be identified.

The main concern regarding ESPB is still a lack of knowledge concerning its mechanism of action. There is no consensus in the literature, and controversial results are even being reported, as in the review study by De Cassai et al. [25]. Possible mechanisms suggested to date by cadaveric, anatomic, and magnetic resonance imaging studies include epidural, paravertebral, intercostal, and extensive lateral and longitudinal diffusion [25]. Based on our current data, we think that epidural spread of LA may contribute to analgesic effect significantly, and we would advise clinicians to consider epidural spread when deciding on the application level and the volume of LA. Further data are still needed for a definite conclusion, but regardless of the 
There may be some technical difficulties in the pediatric population due to anatomical and physiological differences. This patient group has thinner muscle layers, loose connective tissues, and sliding fascial planes. The depth from the skin to the transverse process may also be less than $1 \mathrm{~cm}$, depending on the patient's age. Placing the needle tip immediately beneath the erector spinae muscle may constitute a significant challenge requiring a fine needling technique and a stable patient position. Even though prone positioning appears to be a better option than the lateral position, another difficulty arises since these patients are commonly under general analgesia. Placing the patient in the prone position and repositioning to the supine position may constitute another risk for airway safety. In the case of lumbar ESPB, we overcome these technical and safety concerns by applying the block with the Aksu approach. For thoracic indications, however, we prefer the prone position over the lateral position.

Two complications of ESPB have been reported to date, pneumothorax, and motor weakness [26-27]. Pneumothorax, in our opinion, should be the most unexpected complication where the transverse process represents a natural anatomical barrier between the pleura and needle. Motor weakness following lumbar or low thoracic ESPB with a high volume of LA may be expected due to a widespread to the lumbar plexus. The LA volume used in our clinic is $0.5 \mathrm{ml} / \mathrm{kg}$, limited to a maximum of $20 \mathrm{ml}$ for pediatric patients. According to our records, no motor weakness or block-related complication has been observed to date.

Our results revealed that ESPB has an opioid-sparing effect in pediatric patients, even in major surgeries. However, in addition to the effectiveness of the block, we also think that standardization was one of the major components of adequate pain management achieved. Components of our standard pain management protocol include administering regional techniques before the start of the surgery, giving analgesics at the end of the surgery, evaluating every child with an appropriate pain scoring system according to its age, and administering analgesics based on the pain ladder. We suggest that clinicians adapt their protocols based on their clinical conditions and experiences.

There were some limitations to this study. First, since the data were collected retrospectively and were limited to the case-based experience of a single-center, our results should now be confirmed by future prospective studies. Another limitation was a lack of knowledge concerning the sensory dermal block area of the ESPB. Since all blocks were performed under general anesthesia, it was difficult to determine the exact dermatomal spread and limits of the sensorial block for this group of patients.

\section{Conclusions}

In conclusion, based on our current clinical experience and understanding obtained from the previous literature, ESPB is a safe and effective procedure for achieving opioid-free postoperative analgesia in many different surgical procedures in pediatric patients. While recommending its routine use for pediatric patients, we think that more prospective randomized clinical trials are now needed for a more accurate conclusion concerning its efficiency and safety, and the application levels and LA doses to be used.

\section{Additional Information \\ Disclosures}

Human subjects: Consent was obtained by all participants in this study. Kocaeli Üniversitesi Girişimsel Olmayan Klinik Araştırmalar Etik Kurulu issued approval GOKAEK 2019/85. Local ethics committee (it is also an Institutional Review Board) approval was taken before the start of the study. This study was also registered with clinicaltrials.org (NCT03906019). Animal subjects: All authors have confirmed that this study did not involve animal subjects or tissue. Conflicts of interest: In compliance with the ICMJE uniform disclosure form, all authors declare the following: Payment/services info: All authors have declared that no financial support was received from any organization for the submitted work. Financial relationships: All authors have declared that they have no financial relationships at present or within the previous three years with any organizations that might have an interest in the submitted work. Other relationships: All authors have declared that there are no other relationships or activities that could appear to have influenced the submitted work.

\section{References}

1. Forero M, Adhikary SD, Lopez H, Tsui C, Chin KJ: The erector spinae plane block: a novel analgesic technique in thoracic neuropathic pain. Reg Anesth Pain Med. 2016, 41:621-627. 10.1097/AAP.0000000000000451

2. Schwartzmann A, Peng P, Maciel MA, Forero M: Mechanism of the erector spinae plane block: insights from a magnetic resonance imaging study. Can J Anaesth. 2018, 65:1165-1166. 10.1007/s12630-018-1187-y

3. Adhikary SD, Bernard S, Lopez H, Chin KJ: Erector spinae plane block versus retrolaminar block: a magnetic resonance imaging and anatomical study. Reg Anesth Pain Med. 2018, 43:756-762. 10.1097/AAP.0000000000000798

4. Ivanusic J, Konishi Y, Barrington MJ: A cadaveric study investigating the mechanism of action of erector spinae blockade. Reg Anesth Pain Med. 2018, 43:567-571. 10.1097/AAP.0000000000000789 
5. Gürkan Y, Aksu C, Kuş A, Yörükoğlu UH, Kılıç CT: Ultrasound guided erector spinae plane block reduces postoperative opioid consumption following breast surgery: a randomized controlled study. J Clin Anesth. 2018, 50:65-68. 10.1016/j.jclinane.2018.06.033

6. Tulgar S, Kapakli MS, Şentürk O, Selvi O, Serifsoy TE, Ozer Z: Evaluation of ultrasound-guided erector spinae plane block for postoperative analgesia in laparoscopic cholecystectomy: a prospective, randomized, controlled clinical trial. J Clin Anesth. 2018, 49:101-106. 10.1016/j.jclinane.2018.06.019

7. Aksu C, Şen MC, Akay MA, Baydemir C, Gürkan Y: Erector Spinae Plane Block vs Quadratus Lumborum Block for pediatric lower abdominal surgery: a double blinded, prospective, and randomized trial [Epub ahead of print]. J Clin Anesth. 2019, 57:24-28. 10.1016/j.jclinane.2019.03.006

8. Muñoz F, Cubillos J, Bonilla AJ, Chin KJ: Erector spinae plane block for post- operative analgesia in pediatric oncological thoracic surgery. Can J Anesth. 2017, 64:880-882. 10.1007/s12630-017-0894-0

9. Hernandez MA, Palazzi L, Lapalma J, Forero M, Chin KJ: Erector spinae plane block for surgery of the posterior thoracic wall in a pediatric patient. Reg Anesth Pain Med. 2018, 43:217-219. 10.1097/AAP. 0000000000000716

10. Adhikary SD, Pruett A, Forero M, Thiruvenkatarajan V: Erector spinae plane block as an alternative to epidural analgesia for post-operative analgesia following video-assisted thoracoscopic surgery: a case study and a literature review on the spread of local anaesthetic in the erector spinae plane. Indian J Anaesth. 2018, 62:75-78. 10.4103/ija.IJA_693_17

11. Nardiello MA, Herlitz M: Bilateral single shot erector spinae plane block for pectus excavatum and pectus carinatum surgery in 2 pediatric patients [Article in English, Spanish]. Rev Esp Anestesiol Reanim. 2018, 65:530-533. 10.1016/j.redar.2018.04.006

12. Wyatt K, Elattary T: The erector spinae plane block in a high-risk Ehlers-Danlos syndrome pediatric patient for vascular ring repair. J Clin Anesth. 2019, 54:39-40. 10.1016/j.jclinane.2018.10.031

13. Wong J, Navaratnam M, Boltz G, Maeda K, Ramamurthi RJ, Tsui BCH: Bilateral continuous erector spinae plane blocks for sternotomy in a pediatric cardiac patient. J Clin Anesth. 2018, 47:82-83. 10.1016/j.jclinane.2018.03.020

14. Moore R, Kaplan I, Jiao Y, Oster A: The use of continuous erector spinae plane blockade for analgesia following major abdominal surgery in a one-day old neonate. J Clin Anesth. 2018, 49:17-18. 10.1016/j.jclinane.2018.04.023

15. Aksu C, Gürkan Y: Ultrasound-guided bilateral erector spinae plane block could provide effective postoperative analgesia in laparoscopic cholecystectomy in paediatric patients. Anaesth Crit Care Pain Med. 2019, 38:87-88. 10.1016/j.accpm.2018.03.008

16. Thomas DT, Tulgar S: Ultrasound-guided erector spinae plane block in a child undergoing laparoscopic cholecystectomy. Cureus. 2018, 27:2241. 10.7759/cureus.2241

17. Aksu C, Gürkan Y: Ultrasound guided erector spinae block for postoperative analgesia in pediatric nephrectomy surgeries. J Clin Anesth. 2018, 45:35-36. 10.1016/j.jclinane.2017.12.021

18. Munshey F, Rodriguez S, Diaz E, Tsui B: Continuous erector spinae plane block for an open pyeloplasty in an infant. J Clin Anesth. 2018, 47:47-49. 10.1016/j.jclinane.2018.03.015

19. Aksu C, Gürkan Y: Opioid sparing effect of Erector Spinae Plane block for pediatric bilateral inguinal hernia surgeries. Clin Anesth. 2018, 50:62-63. 10.1016/j.jclinane.2018.06.048

20. Aksu C, Gürkan Y: Aksu approach for lumbar erector spinae plane block for pediatric surgeries . J Clin Anesth. 2019, 54:74-75. 10.1016/j.jclinane.2018.10.043

21. Cesur S, Yayik AM, Öztürk F, Ahiskalioğlu A: Does "Aksu approach" make erector spinae plane block technique easier?. J Clin Anesth. 2019, 55:142-143. 10.1016/j.jclinane.2019.01.010

22. Aksu C, Gürkan Y: Erector spinae plane block: a new indication with a new approach and a recommendation to reduce the risk of pneumothorax. J Clin Anesth. 2019, 54:130-131. 10.1016/j.jclinane.2018.11.007

23. Elkoundi A, Bentalha A, Kettani SEE, Mosadik A, Koraichi AE: Erector spinae plane block for pediatric hip surgery - a case report. Korean J Anesthesiol. 2019, 72:68-71. 10.4097/kja.d.18.00149

24. Baca Q, Lin C, O'Hare K, Golianu B, Tsui B: Erector spinae plane block for pediatric palliative care . Paediatr Anaesth. 2019, 29:386-387. 10.1111/pan.13607

25. De Cassai A, Bonvicini D, Correale C, Sandei L, Tulgar S, Tonetti T: Erector spinae plane block: a systematic qualitative review. Minerva Anestesiol. 2019, 85:308-319. 10.23736/S0375-9393.18.13341-4

26. H Ueshima: Pneumothorax after the erector spinae plane block . J Clin Anesth. 2018, 48:12. 10.1016/j.jclinane.2018.04.009

27. Selvi O, Tulgar S: Ultrasound guided erector spinae plane block as a cause of unintended motor block [Article in English, Spanish]. Rev Esp Anestesiol Reanim. 2018, 65:589-592. 10.1016/j.redar.2018.05.009 